\title{
Impaired postural control in patients affected by tension-type headache
}

\author{
P.G. Giacomini ${ }^{\text {a,* }}$, M. Alessandrini ${ }^{\text {a }}$, M. Evangelista ${ }^{\text {b }}$, B. Napolitano ${ }^{\text {a }}$, \\ R. Lanciani ${ }^{a}$, D. Camaioni ${ }^{b}$ \\ a Otorhinolaryngology Division, University of Rome, "Tor Vergata", Rome, Italy \\ ${ }^{\mathrm{b}}$ Pain Therapy Service, Anesthesiology Institute, Catholic University of the Sacred Heart, Rome, Italy
}

Received 19 August 2003; accepted 2 February 2004

Available online 8 April 2004

\begin{abstract}
Sixteen subjects, affected by chronic tension-type headache (TTH) accordingly to the International Headache Society Classification (1988) criteria, in presence of tenderness in pericranial muscles, with a mean age of $37 \pm 11.8$ years, and ten healthy volunteer subjects, age and sex matched, were submitted to postural analysis by Static Posturography (S.Ve.P. Amplaid). Aim of the study was to evaluate whether patients with TTH have disturbed postural control, as compared to normal subjects. Postural analysis considered all posturographic variables but focused on spectral frequency analysis of body sway. In both open (OE) and closed eyes (CE) condition, spectral frequency analysis showed a significantly increased body sway at low $(\mathrm{OE}=p \leqslant 0.01 ; \mathrm{CE}=p \leqslant 0.01)$ and middle $(\mathrm{OE}=p \leqslant 0.01 ; \mathrm{CE}=p \leqslant 0.01)$ frequencies on the antero-posterior $(y)$ plane and at low frequencies $(\mathrm{OE}=p \leqslant 0.05$; $\mathrm{CE}=p \leqslant 0.05)$ on the lateral $(x)$ plane. Statistical analysis was performed using the Student's $t$ test for unpaired data, $p$ value $\leqslant 0.05$ defined significant.

The proprioceptive input seems to be predominant at middle and high frequencies in maintaining posture, our results seem then to suggest a proprioceptive disturbance in TTH patients. The disturbance is likely related to chronic pericranial muscle contraction and tenderness. Posturography and spectral analysis may help not only in the diagnosis of a postural disturbance but even more in the follow-up of TTH patients, during and after a medical and/or a rehabilitative treatment.

(C) 2004 European Federation of Chapters of the International Association for the Study of Pain. Published by Elsevier Ltd. All rights reserved.
\end{abstract}

Keywords: Tension-type headache; Posturography; Posturography frequency analysis; Dizziness; Muscular contraction

\section{Background}

Tension-type headache (TTH) is the daily or almost daily headache that occurs in a large number of patients who visit medical and neurological practices (Jensen, 2001). Fourteen percent of adults report TTH once or more per week and three percent are affected on a daily or almost daily basis (Schwarts et al., 1998; Jensen, 1999). Females are more frequently affected than males,

\footnotetext{
* Corresponding author. Present address: Policlinico "Tor Vergata", Viale Oxford, 81, 00133 Rome, Italy. Tel.: +39-20902925; fax: +39620902921.

E-mail address: digirolamo@med.uniroma2.it (P.G. Giacomini).
}

and young subjects more frequently affected than older subjects (Schwarts et al., 1998; Jensen, 1999). Despite the fact that TTH is the most common and, as far as socioeconomic impact is concerned, the most important type of headache, remarkably little is known about its pathophysiological background.

Currently, it has been assumed that the muscle factor plays a significant role in the pathophysiological mechanism of TTH which is probably multifactorial: what is known is that more than $80 \%$ of TTH patients have an increased contraction of pericranial muscles (Lipchik et al., 1996) even though in some cases muscle contraction appeared to be a consequence rather than a cause of pain (Jensen et al., 1998). 
There is evidence of a substantial contribution of cervical proprioceptive input to ocular motor control and postural control in both animals and humans: it has been recognized that vestibular, visual and neck proprioceptive information interact in subjective body orientation and mental representation of space (Karnath, 1994). Furthermore, it has been observed that infiltration of local anesthetics into the deep tissues of the neck causes ataxia and nystagmus in animals, but ataxia without nystagmus in humans (Dietrich et al., 1993). Thus, it is reasonable to assume that disturbed cervical proprioception per se affects postural control in humans (Karlberg et al., 1991): in fact it has been reported that patients with chronic cervico-brachial pain and concomitant dizziness manifest impaired postural performance, as compared to healthy subjects (Karlberg et al., 1995).

Postural performance can be assessed objectively by static posturography, recording the forces actuated by subjects feet on the supporting surface. A variety of cues (visual, vestibular and proprioceptive) integrated at higher levels in the CNS, and via the vestibulospinal tract, are needed by leg antigravity muscles to maintain the center of gravity within a body's ground projection.

Static posturography, which involves the analysis of body sway during quiet standing, is a reliable mean of studying the vestibulo-spinal reflex. In previous studies, we devised a mean for quantifying the frequency of body sway in order to analyse more specifically the different sub-systems which contributes to postural control (Giacomini et al., 1998, 2002; Alessandrini et al., 1998, 2003). The spectral frequency analysis consists of evaluating the center of gravity oscillations within a frequency spectrum by a mathematical elaboration according to Fourier. This elaboration of the signal seems able to define different specific postural patterns (in relation to the prevalence of oscillations in pre-established frequency ranges), each of them expressing the predominance of a specific sensorial input.

The aim of the present paper was to study whether patients affected by chronic tension-type headache, in presence of tenderness in pericranial muscles and with no clinical evidence of postural instability, show a disturbed postural control, assessed by means of static posturography and elaborated through the spectral frequency analysis of body sway.

\section{Subjects and methods}

Sixteen consecutive patients (F/M: $12 / 4$, mean age of $37 \pm 11.8$ years) who fulfilled the criteria of IHS classification for primary TTH of chronic type (with over 4 years of disease length) constituted the study group.
Patients were complaining of bilateral pain, localized in the frontal and parietal region, rarely in the occipital region. The quality of pain was dull and pressure-like or tightening and it was described as a sensation of a heavy weight on the top of the head or a ring around the head. The intensity of pain was mild or moderate and it did not restrict the routinary activities or work abilities, it could be triggered out by mental stress and it tended to occur during the stressing situation.

A further inclusion criterium was the presence of tenderness in pericranial muscles Ten healthy volunteer subjects, age and sex matched (F/M: 7/3, mean age of $35 \pm 9.5$ years) free from headache of any kind or muscular tenderness of head and neck area, were the control group. Pericranial muscular tenderness was evaluated, both in patients and controls, by palpation of nine pairs of pericranial muscles and tendon insertions by the physician in a standardized, randomised procedure. The tenderness in each muscle and tendon was scored according to a scale from 0 to 3 , and summed to a Total Tenderness Score (TTS) for each subject as described by Langemark and Olesen (Langemark and Olesen, 1987). All patients showed a TTS above 9 and all controls showed a TTS below 9, equal to the $75 \%$ quartile of TTS from healthy controls: previous studies demonstrated that the ideal cutoff point for separating tension-type headache subjects from non-headache subjects with respect to muscle tenderness was the $75 \%$ quartile of the TTS obtained from a general population (Jensen and Rasmussen, 1996). The exclusion criteria, for both patients and controls, included co-existence of other headache syndromes as migraine or cluster headache; other neurological, systemic (alcohol addiction, thyroid disease, lumbar root disease, etc.), or psychiatric disorders; brain MRI positive for the presence of any intracranial pathology; ingestion of major medications including prophylactics for headache; any form of drug abuse or dependency such as daily ergotamine or large amounts of plain analgesics (on demand, patients were taking non-steroidal anti-inflammatory drugs and/or muscle relaxants); presence of postural hypotension; visual acuity below 10/10; coexistence of labyrinthine symptoms (vertigo or dizziness) or pathological signs at standard vestibular testings (such as spontaneous or evoked nystagmus and abnormal response to the caloric vestibular tests). All patients were examined when free of headache and were not allowed to have taken any analgesics on the day of examination. Familial disposition to TTH was present in $50 \%$ of patients. Population profile is depicted in Table 1.

Static posturography was performed using a normalized computerized static posturography platform (S.Ve.P. Amplaid). Detailed information about the method have been published previously (Giacomini et al., 1998, 2002; Alessandrini et al., 1998, 2003). Briefly, an objective measurement of body sway is ob- 
Table 1

Population profile

\begin{tabular}{llllllll}
\hline Patients & Age & $\begin{array}{l}\text { Quality of } \\
\text { pain }\end{array}$ & $\begin{array}{l}\text { Disease } \\
\text { duration }\end{array}$ & $\begin{array}{l}\text { Prophylactic } \\
\text { therapy }\end{array}$ & $\begin{array}{l}\text { Analgesic } \\
\text { therapy }\end{array}$ & $\begin{array}{l}\text { Headache } \\
\text { syndromes } \\
\text { associated }\end{array}$ & $\begin{array}{l}\text { TTS } \\
\text { Familial } \\
\text { disposition }\end{array}$ \\
\hline 16 & $37 \pm 11.8$ & $\begin{array}{l}\text { Dull and } \\
\text { pressure-like }\end{array}$ & $\begin{array}{l}>4 \text { years } \\
\text { (range: } 4-12 \\
\text { years) }\end{array}$ & None & $\begin{array}{l}\text { NSAIDs and } \\
\text { muscle-relaxants } \\
\text { on demand }\end{array}$ & $\begin{array}{l}\text { No other } \\
\text { headache } \\
\text { associated }\end{array}$ & $>9$ \\
\hline
\end{tabular}

tained using a computer-controlled platform that monitors body sway by three pressure-sensing strain gauges located on the vertexes of an equilateral triangle drawn on the platform.

The subjects are instructed to maintain an upright position during the test, first with eyes open and thereafter with eyes closed. The recording period was $30 \mathrm{~s}$ for each test.

The following parameters may be calculated from the statokinesigram: (a) trace length: the length of the trace made by the center of gravity; (b) trace surface: $90 \%$ of the surface area covered by the trace; (c) mean velocity of body sway: mean velocity of the center of gravity; (d) standard deviation (SD) of velocity of body sway; (e) fast Fourier transformation on $x$ (lateral) and $y$ (anteroposterior) planes (FFTX, FFTY) of the body sway, i.e. the frequency domain analysis of body sway.

Graphic evaluation of spectrograms (two mobile vectors: $x / y$ ) was carried out using Autocad 13 software (Windows/Microsoft). With this procedure, the frequency of body oscillation (power spectra), ranging from 0.01 to $5.00 \mathrm{~Hz}$ (Kohen-Raz et al., 1996), can be quantified.

The frequency spectrum was subdivided into three groups: $0.01-0.70,0.70-1.00$, and $1.00-5.00 \mathrm{~Hz}$. Within each group, the spectral intensity was determined by adding the relative power spectra and the mean $( \pm \mathrm{SD})$ power spectra obtained for each group.

Postural analysis considered all posturographic variables but focused on the FFTX and FFTY results. Neverthless, the specificity of all of these spectral analyses has been shown in our previous studies (Giacomini et al., 1998, 2002).
All results are expressed as mean $\pm \mathrm{SD}$. Statistical analysis of mean differences was performed using the Student's $t$ test for unpaired data (SPSS-Windows), with a $p$ value of 0.05 or less defined as significant.

\section{Results}

Posturography revealed that patients with tensiontype headache exhibit a pattern of body oscillation different from normal subjects.

\subsection{Posturographic variables $(a, b)$}

A significant difference, in patients versus controls, was noticed for both trace length and trace surface, in the closed eyes condition (Table 2).

\subsection{Frequency analysis}

The results of the spectral frequency analysis, in both open and closed eyes condition, are reported in Table 3. A significantly increased body sway was noticed at low and middle frequencies on the antero-posterior $(y)$ plane and at low frequencies on the lateral $(x)$ plane, in both open and closed eyes condition (Figs. 1 and 2).

Table 2

Mean $\pm \mathrm{SD}$ in closed eyes

\begin{tabular}{lll}
\hline & Trace length & Trace surface \\
\hline Patients & $433.2 \pm 182.3^{*}$ & $558.9 \pm 114.1^{*}$ \\
Controls & $302.5 \pm 53.3$ & $252.3 \pm 63.7$ \\
\hline${ }^{*} p<0.05$. & &
\end{tabular}

Table 3

Results of the spectral frequency analysis, in both open and closed eyes condition

\begin{tabular}{llllllll}
\hline & & $X$ I & $X$ II & $X$ III & $Y$ I & $Y$ II & $Y$ III \\
\hline Patients & OE & $22.1 \pm 4.1^{* *}$ & $4.95 \pm 2.2$ & $1.2 \pm 1.0$ & $21.2 \pm 3.9^{* *}$ & $8.1 \pm 4.9^{* *}$ & $0.9 \pm 0.5$ \\
& CE & $23.0 \pm 1.6^{*}$ & $6.5 \pm 2.0$ & $2.7 \pm 0.7$ & $20.3 \pm 4.9^{* *}$ & $12.1 \pm 9.3^{* *}$ & $2.5 \pm 0.9$ \\
Controls & OE & $14.6 \pm 0.6$ & $4.2 \pm 0.7$ & $1.3 \pm 1.0$ & $14.3 \pm 1.2$ & $3.5 \pm 0.7$ & $1.1 \pm 0,7$ \\
& CE & $18.6 \pm 2.1$ & $5.8 \pm 1.4$ & $3.0 \pm 0.8$ & $12.8 \pm 2.2$ & $3.5 \pm 1.4$ & $2.4 \pm 1.1$ \\
\hline
\end{tabular}

$\mathrm{OE}=$ open eyes condition; $\mathrm{CE}=$ closed eyes condition; $X=$ mean $\pm \mathrm{SD}$ of the power spectra on $x$ plane; $Y=$ mean $\pm \mathrm{SD}$ of the power spectra on $y$ plane; $\mathrm{I}=$ frequency range $0.01-0.7 \mathrm{~Hz} ; \mathrm{II}=$ frequency range $0.7-1.0 \mathrm{~Hz}$; III = frequency range $1.0-5.0 \mathrm{~Hz}$.

${ }^{*} p<0.05$.

** $p<0.01$. 


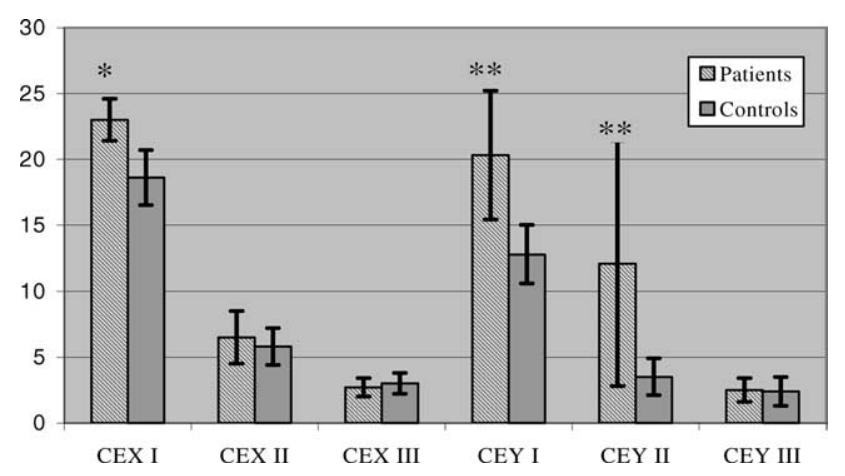

Fig. 1. Power spectra in patients vs. controls, in closed eyes (CE) condition. $* p<0.05, * * p<0.01$.

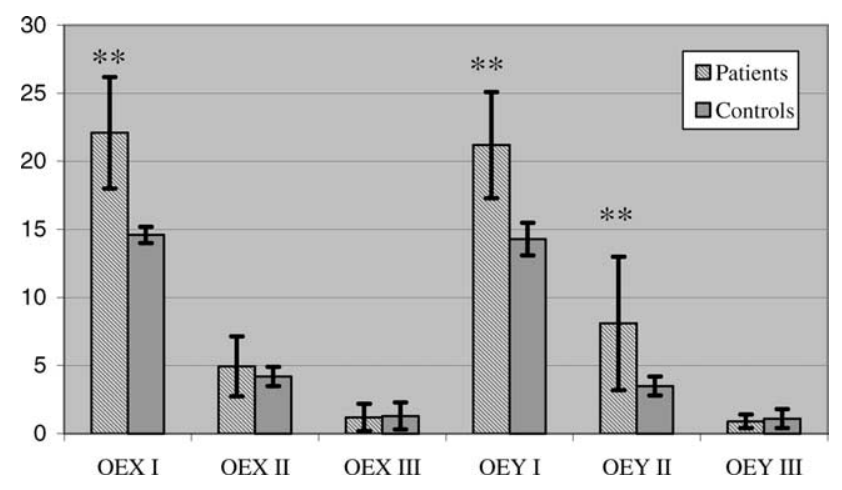

Fig. 2. Power spectra in patients vs. controls, in open eyes (OE) condition. $* p<0.05, * * p<0.01$.

\section{Conclusion}

Patients affected by TTH often complain of dizziness, unsteadiness or disequilibrium. Patients report on a sensation of "floating" and gait deviation both while standing and while walking (Brandt, 1996; Hulse and Holzl, 2000) rather than the typical rotatory vertigo.

In this paper, we hypothesize that a disturbed postural control in TTH patients might be in relation with altered signals from the pericranial muscles, chronically tender and contracted.

Previous papers described not only the association of dizziness and cervical pain syndromes of different origin, but also the relation of the impaired postural performance with an abnormal proprioceptive signals rather than with a vestibular dysfunction (Revel et al., 1991, 1994).

Balance function subserves two goals: stabilization of the visual field and achievement of the upright position. The upright stance is the result of the complex integration of peripheral sensory inputs, from visual, proprioceptive and posterior labirinth apparatus, at different levels in CNS (brainstem, cerebellum, basal ganglia and thalamus).

Analysis of posture by static posturography provides information on overall postural performance with variables such as trace length, surface and velocity of body sway. It has been suggested that the labirinth is a main monitor of the surface of body sway, while proprioception is a principle monitor of the velocity of body movement of sway (Nagakawa et al., 1993; Yasuda et al., 1999). Specifically, frequencies of body sway are analyzed and quantified by fast Fourier transformation (Yoneda and Tokumasu, 1986). Spectral frequency analysis of body sway seems to identify ranges of low, middle and high frequencies which are thought to be influenced in varying degrees by different sensory inputs. Low frequencies are mainly under vestibular control, while higher frequencies are guided by proprioceptive information (Kohen-Raz et al., 1996). Proprioception seems to control mainly the antero-posterior sway when vestibular inputs are maintained (Nagakawa et al., 1993; Giacomini et al., 2002).

In TTH patients, the posture is grossly maintained, but an increase of descriptive posturographic parameters such as trace length and surface under the closed eyes condition, suggests a greater effort (i.e. use of visual support) needed to achieve this goal. The visual dependence of TTH patients seems to be increased: we felt it may be a compensative effect aimed to counterbalance the misleading effect of abnormal proprioceptive input.

Spectral analysis of body sway shows a significantly increased body sway at low and middle frequencies on the antero-posterior $(y)$ plane and at low frequencies on the lateral $(x)$ plane, in both open and closed eyes condition. As proprioceptive control seems to manifest mainly on middle frequencies and on sagittal plane (Giacomini et al., 1998): the present results would suggest that in TTH patients a proprioceptive disturbance may account for the altered postural pattern and for the dizzy symptomatology. Static posturography has still little localizing value with regard to vertigo. Some exception are idiopathic postural tremor (Yarrow et al., 2001) or cerebellar pathology (Diener et al., 1984). Posturography spectral frequency analysis revealed an altered postural pattern specific for TTH patients, suggesting the hypothesis that increased muscular tension, as measured by TTS score, sensitizes the proprioceptors, resulting in an erroneous input from neck muscles: its convergence with correct visual and vestibular information, at the level of CNS, might produce a sensory mis-match, responsible of the sensation of disequilibrium (Johansson and Sojka, 1991). Alternative explanations of such a phenomenon may involve attentional deficits or different breathing patterns: the former were not grossly noted in our cases nor were the patients submitted to drugs interfering with attention, the latter were not studied in our cases therefore no definitive conclusions may be drown about it.

The posturography findings of the present study, in patients otherwise normal at the standard otoneurological examination (audio-vestibular testing and brain MRI), seem worth of further confirmation by larger series 
and neurophysiological tests. Furthermore, we believe that, in clinical practice, posturographic results might help not only in the diagnosis of a postural disturbance, but even more in the follow-up of patients, revealing any change of the patient postural pattern during and after the medical and/or rehabilitative treatment.

\section{References}

Alessandrini M, Giacomini P, Sorace F, Bruno E. Postural stabilization after otholitic repositioning for posterior semicircular canal canalolithiasis. Acta Otorhinolaryngol Ital 1998;18:368-72.

Alessandrini M, D’Erme G, Bruno E, Napolitano B, Magrini A. Vestibular compensation: analysis of postural re-arrangement as a control index for unilateral vestibular deficit. Neuroreport 2003;14(7):1075-9.

Brandt T. Cervical vertigo. Reality or fiction. Audiol-Neurotol 1996;1(4):187-96.

Diener HC, Dichgans J, Bacher M, Gompf B. Quantification of postural sway in normals and patients with cerebellar diseases. Electroencephalogr Clin Neurophysiol 1984;57(2):134-42.

Dietrich M, Pollmann W, Pfaffenrath V. Cervicogenic headache: electronystagmography, perception of verticality and posturography in patients before and after C2-blockade. Cephalalgia 1993;13(4):285-8

Giacomini P, Sorace F, Magrini A, Alessandrini M. Spectral analysis in stabilometry for alterations in postural control. Acta Otorhinolaryngol Ital 1998;18:83-7.

Giacomini PG, Alessandrini M, Magrini A. Long term postural abnormalities in benign paroxysmal positional vertigo. ORL J Otorhinolaryngol Relat Spectrosc 2002;64:237-41.

Hulse M, Holzl M. Vestibulospinal reactions in cervicogenic disequilibrium. Cervicogenic imbalance. HNO 2000;48(4):295-301.

Johansson H, Sojka P. Pathophysiological mechanisms involved in genesis and spread of muscular tension in occupational muscle pain and in chronic musculoskeletal pain syndromes: a hypothesis. Med Hypotheses 1991;35:196-203.

Jensen R, Rasmussen BK. Muscular disorders in tension-type headache. Cephalalgia 1996;16:97-103.

Jensen R, Bendtsen L, Olesen J. Muscular factors are of importance in tension-type headache. Headache 1998;38:10-7.
Jensen R. Pathophysiological mechanisms of tension-type headache: a review of epidemiological and experimental studies. Cephalalgia 1999; 19:602-21.

Jensen R. Mechanism of tension-type headache. Cephalalgia 2001;21(7):786-9.

Karlberg M, Magnusson M, Johansson R. Effects of restrained cervical mobility on voluntary eye movements and postural control. Acta Otolaryngol (Stockh) 1991;111:664-70.

Karlberg M, Persson L, Magnusson M. Impaired postural control in patients with cervico-brachial pain. Acta Otolaryngol (Stockh) 1995;(Suppl 520):440-2.

Karnath H-O. Subjective body orientation in neglect and the interactive contribution of muscle proprioception and vestibular stimulation. Brain 1994;117:1001-12.

Kohen-Raz R, Himmelfarb M, Tzur S, Shub Y. An initial evaluation of fatigue and circadian changes as assessed by multiple posturography. Percept Mot Skills 1996;82:547-77.

Langemark M, Olesen J. Pericranial tenderness in tension headache: a blind controlled study. Cephalalgia 1987;7:249-55.

Lipchik GL, Holroyd KA, France CR, Kvaal SA, Segal D, Cordingley GE, et al. Central and peripheral mechanisms in chronic tensiontype headache. Pain 1996;64:467-75.

Nagakawa H, Ohashi N, Watanabe Y, Mizukoshi K. The contribution of proprioception to postural control in normal subjects (Stockh). Acta Otolaryngol 1993;(Suppl 504):112-6.

Revel M, Andre-Deshays C, Minguet M. Cervicocephalic kinesthetic sensibility in patients with cervical pain. Arch Phys Med Rehabil 1991;72(5):288-91.

Revel M, Minguet M, Gergory P, Vaillant J, Manuel JL. Changes in cervicocephalic kinestesia after a proprioceptive rehabilitation program in patients with neck pain: a randomized controlled study. Arch Phys Med Rehabil 1994;75: 895-9.

Schwarts BS, Stewart WF, Simon D, Lipton RB. Epidemiology of tension-type headache. JAMA 1998;279(5):381-3.

Yarrow K, Brown P, Gresty MA, Bronstein AM. Force platform recordings in the diagnosis of primary orthostatic tremor. Gait Posture 2001;13(1):27-34.

Yasuda T, Nagakawa T, Inoue H, Iwamoto M, Inokuchi A. The role of the labyrinth, proprioception and plantar mechanosensor in the manteinance of an upright posture. Eur Arch Otorhinolaryngol 1999;256:S27-32.

Yoneda S, Tokumasu K. Frequency analysis of body sway in the upright posture. Acta Otolaryngol 1986;102:87-92. 